\title{
The Trojan Horse Method for nuclear astrophysics and its recent applications
}

L. Lamia ${ }^{1,2, \star}$, C. Spitaleri ${ }^{1,2}$, M. Mazzocco ${ }^{3,4}$, A. Boiano ${ }^{6}$, C. Boiano ${ }^{13}$, C. Broggini ${ }^{4}$, A. Caciolli ${ }^{3,4}$, R. Depalo ${ }^{3,4}$, A. Di Pietro ${ }^{2}$, P. Figuera ${ }^{2}$, F. Galtarossa ${ }^{15}$, G.L. Guardo ${ }^{1,2}$, M. Gulino ${ }^{1,10}$, S. Hayakawa $^{7}$, S. Kubono 8,14 , M. La Cognata ${ }^{1}$, M. La Commara ${ }^{6,9}$, G. La Rana ${ }^{6,9}$, M. Lattuada ${ }^{1,2}$, R. Menegazzo $^{4}$, A. Pakou ${ }^{5}$, C. Parascandolo ${ }^{6}$, D. Piatti ${ }^{3,4}$, D. Pierroutsakou ${ }^{6}$, R.G. Pizzone ${ }^{1}$, S.M.R. Pugliaa $^{1,2}$, S. Romano ${ }^{1,2}$, G.G. Rapisarda ${ }^{1}$, A.M. Sanchez-Benitez ${ }^{17}$, M.L. Sergi ${ }^{1}$, O. Sgouros ${ }^{5}$, H. Silva $^{18}$, F. Soramel ${ }^{3,4}$, V. Soukeras ${ }^{5}$, E. Strano ${ }^{3,4}$, D. Torresi ${ }^{1}$, O. Trippella ${ }^{19,20}$, A. Tumino ${ }^{1,10}$, H. Yamaguchi $^{7}$, F.L. Villante ${ }^{11,12}$, and G.L. Zhang ${ }^{16}$

${ }^{1}$ Laboratori Nazionali del Sud di Catania, LNS-INFN, Italy

${ }^{2}$ Dipartimento di Fisica e Astronomia, Università di Catania, Italy

${ }^{3}$ Dipartimento di Fisica e Astronomia, Università di Padova, Italy

${ }^{4}$ INFN-Sezione di Padova, Italy

${ }^{5}$ Department of Physics and HINP, University of loannina, Greece

${ }^{6}$ INFN-Sezione di Napoli, Italy

${ }^{7}$ Center for Nuclear Study, University of Tokyo, Japan

${ }^{8}$ Riken Nishina Center, Wako, Saitama, Japan

${ }^{9}$ Dipartimento di Scienze Fisiche, Università di Napoli, Italy

${ }^{10}$ Facoltà di Ingegneria e Architettura, Università degli Studi di Enna Kore, Italy

${ }^{11}$ Laboratori Nazionali del Gran Sasso LNGS-INFN, Assergi (AQ), Italy

${ }^{12}$ Dipartimento di Scienze Fisiche e Chimiche, Università de L'Aquila, Italy

${ }^{13}$ INFN-Sezione di Milano, Italy

${ }^{14}$ Institute of Modern Physics, Chinese Academy of Sciences, China

${ }^{15}$ Laboratori Nazionali di Legnaro, LNL-INFN, Italy

${ }^{16}$ School of Physics and Nuclear Energy Engineering, Bihang University

${ }^{17}$ Universitad de Huelva, Spain

${ }^{18}$ Universitad de Lisboa, Purtugal

${ }^{19}$ Dipartimento di Fisica e Geologia, Università di Perugia, Italy

${ }^{20}$ INFN-Sezione di Perugia, Italy

\footnotetext{
^e-mail: 1lamia@lns.infn.it
} 


\section{Introduction}

Experimental nuclear astrophysics aims at measuring nuclear reaction cross sections of interest for both primordial and stellar nucleosynthesis in the corresponding Gamow windows. Often extrapolations from high energy data down to Gamow energies are used, although they could suffer of uncertainties related, for instance, to the presence of unknown low-lying (or even subthreshold) resonances or to the electron screening phenomenon [1,2]. This effect, due to the electron (atomic) cloud surrounding the positively charged nucleus, alters the cross-section at low-energies, thus partially shielding the pure Coulomb repulsion between the charged-interacting nuclei. Electron screening understanding is far to be completely achieved since the current available theoretical dynamics models (i.e. adiabatic approximation) largely underestimate the electron screening potential values with respect to those measured in terrestrial laboratories.

\section{The Trojan Horse Method}

The Trojan Horse Method (THM) [3-6] allows one to extract the bare-nucleus cross-section of a charged-particle induced reaction $\mathrm{a}+\mathrm{x} \rightarrow \mathrm{c}+\mathrm{C}$ at astrophysical energies free of Coulomb suppression, by properly selecting the quasi-free $(\mathrm{QF})$ contribution of an appropriate reaction $\mathrm{a}+\mathrm{A} \rightarrow \mathrm{c}+\mathrm{C}+\mathrm{s}$, performed at energies well above the Coulomb barrier, where the nucleus $\mathrm{A}$ has a dominant $\mathrm{x} \oplus \mathrm{s}$ cluster configuration. The $\mathrm{a}+\mathrm{x} \rightarrow \mathrm{c}+\mathrm{C}$ reaction studied with $\mathrm{THM}$ is not affected by tunneling, since the $a+x$ interaction occurs in the pure nuclear field without the influence of the Coulomb barrier. This allows to the experimentalist to measure the corresponding $\mathrm{S}(\mathrm{E})$-factor even at energies at which direct measurements can access only via the extrapolations. However, it must be stressed here that the $\mathrm{TH} \mathrm{S}(\mathrm{E})$-factor determination requires a normalization procedure to the available direct measurements performed at higher energies, thus rendering the THM a complementary experimental technique for nuclear astrophysics aimed at reaching the ultra-low energy region of interest for astrophysical applications. THM has been used in studying several problems, ranging from BBN [7], light element burning reactions [8-12], CNO reactions [13-15], explosive nucleosynthesis involving RIB $[16,17]$ and removing/producing neutron reactions $[18,19]$.

\subsection{Some details on THM data analysis}

The THM can be regarded as an extension to the ultra low-energies of QF reactions, widely used in the past for evaluating nuclear structure with particular regard to the cluster configurations, making it possible to apply the method to nuclear astrophysics [4, 20,21]. By performing a devoted experiment for studying the three-body reaction $\mathrm{a}+\mathrm{A} \rightarrow \mathrm{c}+\mathrm{C}+\mathrm{s}$, it is possible to connect the three-body cross section with the one of interest for astrophysics through the relation $[5,6]$

$$
\left.\frac{d^{3} \sigma}{d E_{c} C \Omega_{c} C \Omega_{C}} \propto K F \cdot\left|\Phi\left(\overrightarrow{p_{s}}\right)\right|^{2} \cdot\left(\frac{d \sigma}{d \Omega}\right)\right|_{a-x} ^{H O E S}
$$

where $K F$ represents the kinematical factor, $\left|\Phi\left(\overrightarrow{p_{s}}\right)\right|^{2}$ is the square of the momentum distribution for the $x-s$ relative motion inside the TH-nucleus $A$, and $\left.\frac{d \sigma}{d \Omega}\right|_{a-x} ^{H O E S}$ the half-off energy shell cross section. This last quantity represents the "bare-nucleus" cross section of interest for astrophysics, that needs to be corrected for the penetrability through the Coulomb barrier and normalized to the available 
high-energy direct data. More advanced approaches have been introduced in later years, allowing us to justify and generalize Eq. 1, releasing some approximations contained in it (see, for instance, $[5,6,15,19])$. In order to assess the method, some of the most important sources of uncertainties in a typical THM experiment and/or data analysis need to be underlined:

- Energy resolution effects. THM data are expressed in terms of the relative energy between twoout-of three $c$ and $C$ detected particles, being their detection sufficient for reconstructing completely the full kinematic of a three-body exit channel reaction by applying energy and momentum conservation rules. Energy resolution effects on the relative $\mathrm{E}_{c C}$ energy can be evaluated via the standard errors propagation theory, taking into account both energy and angular resolution due to the adopted experimental setup. For instance, in the case of the ${ }^{11} \mathrm{~B}+\mathrm{p}$ reaction discussed in $[22,23]$, this has been evaluated to be $\sim 40 \mathrm{keV}$;

- Determination of the experimental momentum distribution. The experimental determination of the momentum distribution for the intercluster $x-s$ motion is one of the most important steps of a typical THM analysis, this reflecting the presence of the quasi-free reaction mechanism. Its behavior is then evaluated in terms of both Plane Wave Impulse Approximation (PWIA) and Distorted Wave Born Approximation (DWBA). By referring to the recent ${ }^{10} B+p$ case $[24,25]$, its has been found that the two approaches, PW and DWBA, nicely agree within lower momentum of the exiting neutron, being the same result confirmed in a variety of THM experiments discussed in the literature (see [13] for instance);

- Correction for barrier penetration effects. THM cross section data are not affectced by barrier penetration effects. For charged particle-induced reactions, one has to take into account Coulomb as well as centrifugal effects while in the case of neutron-induced reactions only the latter need to be considered. Thus, THM data need to be corrected for an analytical function describing the penetration through the barrier, as given in several text books, i.e. [1]. For such purposes, ones has to fix a cut-off radius in terms of the standard formula $r=1.2 *\left(A_{1}^{1 / 3}+A_{2}^{1 / 3}\right) \mathrm{fm}$ [1], even if such choice introduces systematic uncertainties on the final results. This further source of uncertainty has been investigated in THM papers such as [23], for which an overall uncertainty of $\sim 14 \%$ on the final evaluation of the zero-energy $\mathrm{S}(\mathrm{E})$-factor has been found;

- Influence of the momentum distribution in THM data. The role of the momentum distribution and, in particular, of the FWHM of the experimental momentum distribution on THM data has been investigated in [27], where we have evaluated the role of the $d$-state when describing the deuteron ground-state wave function. In particular, both the $s$ and $d$ state wave functions have been calculated by using the exact form and the asymptotic one, their shape being the one shown in the left panel of Fig.1. In order to evaluate the impact on THM data, we have introduced the $d$ state contribution in the THM analysis of [23] and we found a maximum variation of about $0.5 \%$ on the absolute value of the $S(0)$ for the ${ }^{11} \mathrm{~B}+\mathrm{p}$ reaction.

\section{The recent ${ }^{7} \mathrm{Be}(\mathrm{n}, \alpha)^{4} \mathrm{He}$ THM investigation}

To shed light on the cosmological lithium-problem of interest in the Big Bang Nucleosynthesis (BBN), nuclear physic solutions have been extensively investigated $[7,28]$. Among the nuclear reactions producing or destroying lithium, the ones involving the radioactive ${ }^{7} \mathrm{Be}$ isotope $\left(\mathrm{t}_{1 / 2}=53.22 \pm 0.06 \mathrm{~d}\right)$ and neutrons have been matter of recent studies aimed at investigating the (n,p) and (n, $\alpha)$ reaction channels at Gamow energies (about $100 \mathrm{keV}$ ) [29, 30]. To complement such information, the Trojan Horse Method (THM) appears to be suitable since it allows us to study neutron-induced reactions with unstable beams overcoming all the experimental difficulties related to the direct measurements of such 

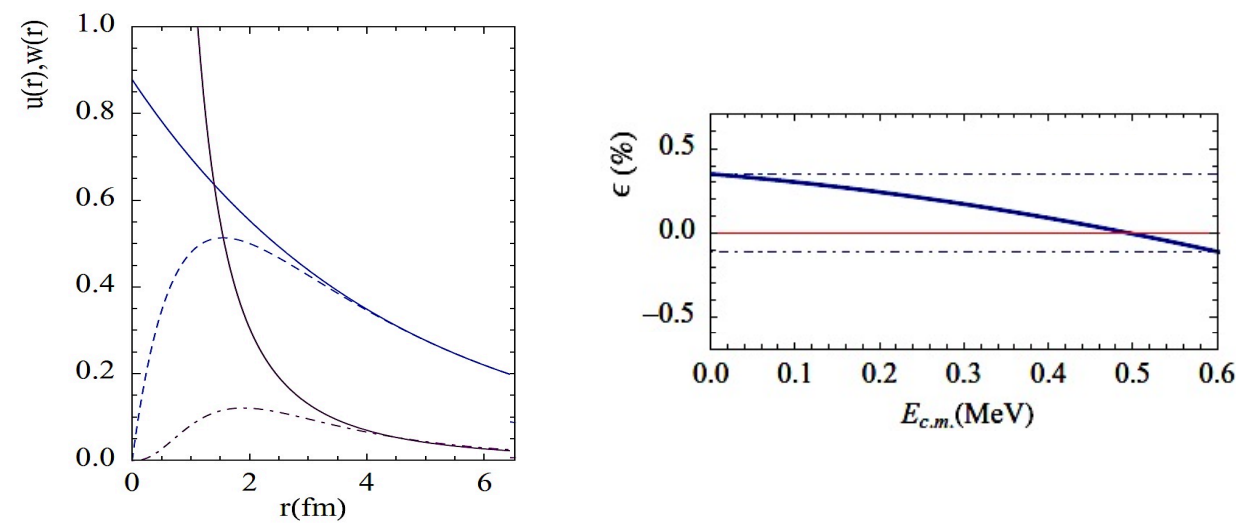

Figure 1. (Left panel). Deuteron ground state wave function: the exact (dashed) and asymptotic (full line) solution for the $s$-state $(u(r)$, blue color) and the $d$-state $(w(r)$, purple color). (Right panel). Influence of the $d$-state component of the deuteron wave function on the ${ }^{11} \mathrm{~B}-\mathrm{p}$ THM analysis, leading to a maximum variation of $0.5 \%$ [27].

processes [5, 6]. To this aim, a devoted experimental research program started in 2015 in order to study the ${ }^{7} \mathrm{Be}-\mathrm{n}$ induced reactions by means of THM with two independent measurements performed at the Radioactive Ion Beam in-flight facility EXOTIC [31, 32] INFN-LNL (Legnaro, Italy) and at at Center-for-Nuclear-Study Radioactive Isotope Beam (CRIB) (Riken, Japan). The preliminary results of the CRIB experiment are discussed in this volume (see contribution from S. Hayakawa in this volume).

In general, the ${ }^{7} \mathrm{Be}(\mathrm{n}, \alpha)^{4} \mathrm{He}$ reaction $(\mathrm{Q}=18.99 \mathrm{MeV})$ has been studied by means of the THM applied to the quasi-free reaction ${ }^{2} \mathrm{H}\left({ }^{7} \mathrm{Be}, \alpha^{4} \mathrm{He}\right) \mathrm{p}(\mathrm{Q}=16.765 \mathrm{MeV})$, by using a $20.4 \mathrm{MeV}{ }^{7} \mathrm{Be}$ beam impinging on a $400 \mu \mathrm{g} / \mathrm{cm}^{2}$ thick $\mathrm{CD}_{2}$ target. By referring to the polar diagram of Fig.2 (left panel), deuteron has been used as TH-nucleus, undergoing its break-up in neutron (participant) and proton (spectator). Additionally, only quasi-free (QF) events are the ones of interest for THM analysis, corresponding to the kinematical conditions for which, in the exit channel, proton maintains the same momentum distribution it had inside the deuteron before the occurrence of break-up [5, 6]. For the purposes of the experiment, only the two emerging alpha particles have been detected while the kinematical quantities of the undetected proton have been reconstructed by means of momentum-energy conservation laws. The BELICOS (BEryllium and LIthium in the COSmos) experiment has been performed at the facility EXOTIC, where the production of the unstable ${ }^{7} \mathrm{Be}$ beam has been already accomplished in the past. In the present case, the ${ }^{7} \mathrm{Be}$ beam has been produced by means of a $33 \mathrm{MeV}{ }^{7} \mathrm{Li}$ beam interacting with a 1 bar $\mathrm{H}_{2}$ cryogenic gas target. At the end of the beam line, an intensity of $5-8 \cdot 10^{5} \mathrm{pps}$ and a purity of about $99 \%$ were measured. The adopted experimental setup for the present ${ }^{7} \mathrm{Be}+{ }^{2} \mathrm{H}$ experiment is sketched in Fig.2 (right panel). It is part of the EXPADES array described in [33]. The detectors have been located around the so-called QF angular pairs, i.e. the angular pairs at which alpha particles are emitted in correspondence of low-momenta of the undetected proton, thus completely covering the kinematic region at which the contribution of the QF reaction mechanism is expected to be dominant. A symmetrical configuration of the detection system has been chosen to double statistics. The alpha particles emitted in the angular range $27^{\circ} \pm 8^{\circ}$ have been detected by means of a $\Delta \mathrm{E}-\mathrm{E}$ telescope made up of an ionization chamber (IC), ( $\Delta \mathrm{E}$ stage), and two $300 \mu \mathrm{m}$ silicon detectors acting as $\mathrm{E}$ stage (T2 

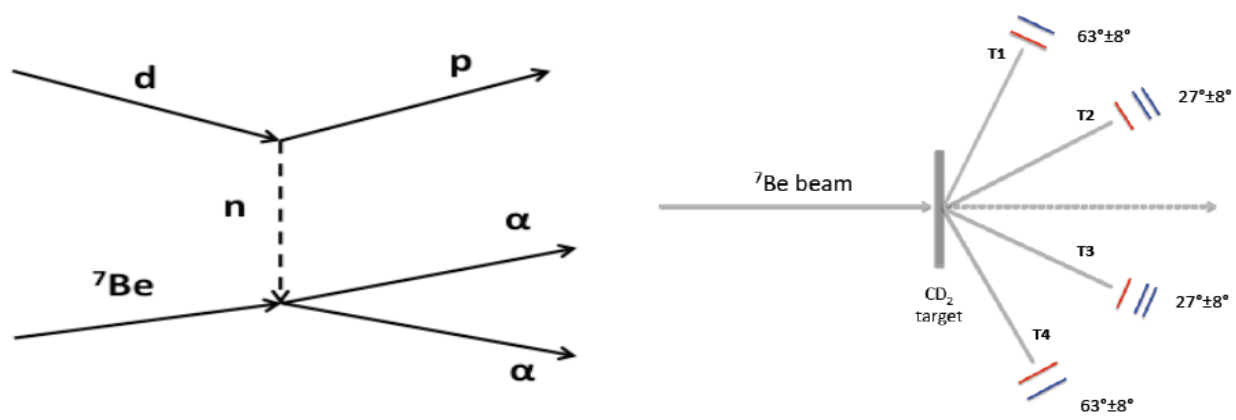

Figure 2. (Left panel). The polar diagram describing the quasi-free $7 \mathrm{Be}+\mathrm{d}$ interaction. The lower pole describes the ${ }^{7} \mathrm{Be}-\mathrm{n}$ interaction of interest.(Right panel). The experimental setup adopted for the BELICOS experiment.

and T3, in Fig.2). The IC have had an active depth of $61.5 \mathrm{~mm}$ and have been filled with $100 \mathrm{mbar}$ isobutane gas. Entrance and exit windows were made up by $1.5 \mu \mathrm{m}$ thick mylar foils with an effective area of $60 \times 60 \mathrm{~mm}^{2}$ to match the E silicon-stage. The further stage of the analysis foresees the selection of the events corresponding to the three-body reaction channel ${ }^{2} \mathrm{H}\left({ }^{7} \mathrm{Be}, \alpha^{4} \mathrm{He}\right) \mathrm{p}$. The coincidence events between T2-T4 and T1-T3 have been studied. By using the standard $\Delta \mathrm{E}-\mathrm{E}$ technique to select the $\mathrm{Z}=2$ loci in the telescopes, the alpha-alpha events of interest have been reconstructed once the energy loss in the $\mathrm{CD}_{2}$ target as well as in the IC has been properly evaluated. To assess the proper selection of the exit channel the experimental Q-value spectrum has been deduced for the selected events (Fig.3, left panel). The experimental peak is centered at an energy of about $16.76 \mathrm{MeV}$, in agreement with the theoretical one of $16.765 \mathrm{MeV}$. A Gaussian fit of such a peak leads to a FWHM of about $2 \mathrm{MeV}$, reflecting the experimental FWHM of the ${ }^{7} \mathrm{Be}$ beam (FWHM $\approx 1 \mathrm{MeV}$ ), energy loss effects in the $\mathrm{CD}_{2}$ target $(\approx 0.7 \mathrm{MeV})$ and angular resolution $\left( \pm 0.4^{\circ}\right)$ effects. In order to select the QF-reaction mechanism, on which the full THM data analysis is based, the trend of the momentum distribution for the $\mathrm{p}-\mathrm{n}$ intercluster motion inside deuteron has been studied and the preliminary result is shown in the right panel of Fig.3 (black dots). The red-curve of Fig.3 represents the theorectial momentum distribution for the p-n intercluster motion usually described in terms of the squared modulus of the Hulthén wave function in mometum space. The agreement between our data and the theoretical distribution is a strong evidence of the presence of the QF-reaction mechanism thus allowing us to further proceed in the extraction of the ${ }^{7} \mathrm{Be}(\mathrm{n}, \alpha)^{4} \mathrm{He}$ cross section. The data analysis is still on going.

\section{References}

[1] Rolfs, C. \& Rodney W. 1988, Cauldrons in the Cosmos (The Univ. of Chicago, Chicago)

[2] Spitaleri C., Bertulani C.A., Fortunato L. et al. 2016, Phys. Lett. B, 755, 275-278

[3] G. Baur 1986, Phys. Lett., 178, 135

[4] Spitaleri C. et al., Problems of Fundamental Modern Physics, edited by R. Cherubini, P. Dalpiaz, and B. Minetti (World Scientific, Singapore, 1991), p. 211.

[5] Tribble R.E., Bertulani C.A., La Cognata M. et al. 2014, Rep. Prog. Phys., 77, 106901

[6] Spitaleri C., La Cognata M., Lamia L. et al. 2016, Eur. Phys. J. A, 52, 77

[7] Pizzone R.G., R. Spartà, Bertulani C.A. et al. 2014, The Astrophys. Journ., 786, 112 

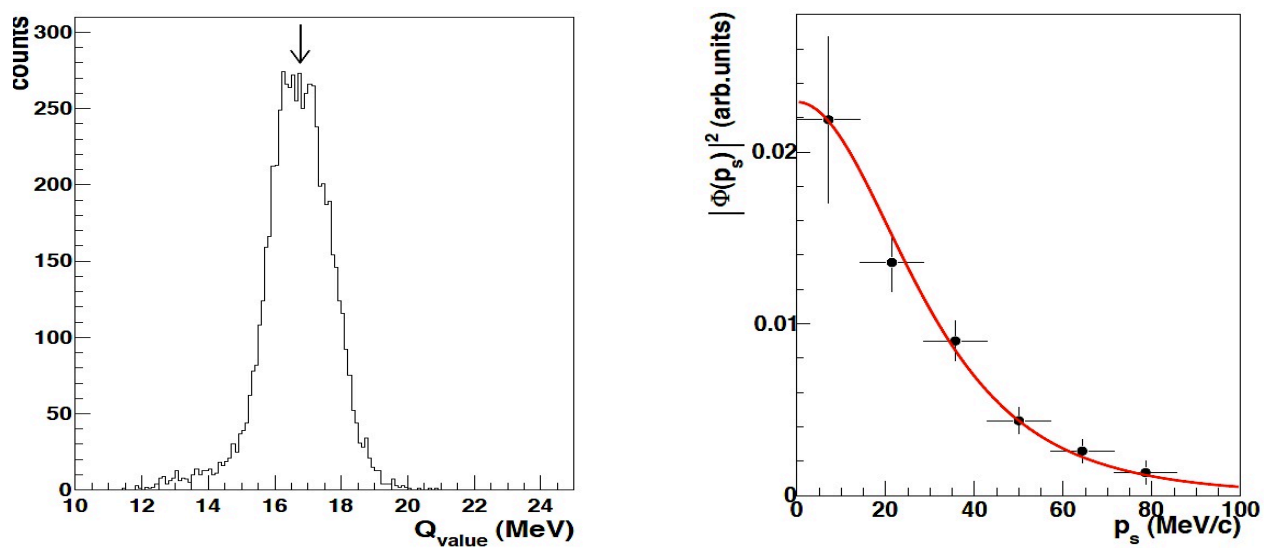

Figure 3. (Left panel). The experimental Q-value spectrum for the selected ${ }^{2} \mathrm{H}\left({ }^{7} \mathrm{Be}, \alpha^{4} \mathrm{He}\right) \mathrm{p}$ events. The arrow marks the position of the theoretical value. No other competing channels are detectable. (Right panel). The preliminary experimental momentum distribution (black dots) compared with the theoretical one (red line) give in terms of the modulus square of the Hulthén wave function in momentum space.

[8] Tumino A., Spitaleri C., Mukhamedzhanov A. et al. 2011, Phys.Lett. B, 705, 546

[9] Pizzone R.G., Spitaleri C., Bertulani C.A. et al. 2013, Phys. Rev. C, 87, 025805

[10] Lamia L., Spitaleri C., Tognelli E. et al. 2015, The Astrophys. Journ., 811, 99

[11] Chengbo Li, Qungang Wen, Yuanyong Fu et al. 2015, Phys. Rev. C, 92, 025805

[12] Grineviciute J., Lamia L., Mukhamedzhanov A. et al. 2015, Phys. Rev. C, 91, 014601

[13] Sergi M.L., Spitaleri C., La Cognata M. et al. 2015, Phys.Rev.C, 91, 065803

[14] Palmerini S., Sergi M.L., La Cognata M. et al. 2013, The Astrophysical Journal, 764, 128

[15] La Cognata M., Spitaleri C., Trippella O. et al. 2013, The Astr. Phys. Journ., 777, 143

[16] Cherubini S., Gulino M., Spitaleri C. et al. 2015, Phys., Rev. C, 92, 015805

[17] Pizzone R.G., Roederer B., McCleskey M. et al. 2016, EpJ A, 52, 24

[18] Gulino M., Spitaleri C., Tang X.D. et al. 2013, Phys. Rev. C, 87, 012801

[19] Guardo G.L., Spitaleri C., Lamia L. et al., 2017, Phys. Rev. C, 95, 025807

[20] Zadro M., Miljanic D., Spitaleri C. et al. 1989, Phys. Rev. C, 40, 181

[21] Calvi G., Lattuada M., Miljanic D. et al., 1990, Phys. Rev. C, 41, 1848

[22] Spitaleri C., Lamia L., Tumino A. et al. 2004, Phys.Rev.C, 69055806

[23] Lamia L., Spitaleri C., Carlin N. et al. 2012, J. Phys. G, 39, 015106

[24] Spitaleri C., Lamia L., Puglia S.M.R. et al. 2014 Phys.Rev.C 90035801

[25] Spitaleri C., Puglia S.M.R., La Cognata M. et al. 2017 Phys.Rev.C 95035801

[26] Angulo, C. et al. 1999, Nucl. Phys. A, 656, 3

[27] Lamia L., La Cognata M., Spitaleri C. et al. 2012, Phys. Rev. C, 85, 025805

[28] Broggini C., Canton L., Fiorentini G. et al. 2012, J. Cosmol. Astropart. Phys., 6, 30

[29] Barbagallo M., Musumarra A., Cosentino L. et al. 2016, Phys. Rev. Lett., 117, 152701

[30] Kawabata T., Fujikawa Y., Furuno T. et al. Phys. Rev. Lett. 2017, 118, 052701

[31] Maidikov V.Z. et al. 2004, Nucl. Phys. A, 746, 389c 
[32] Farinon F., Glodariu T., Mazzocco M. et al. 2008, Nucl. Instr. and Meth. B, 266, 4097

[33] Pierroutsakou D., Boiano A., Boiano C. et al. 2016, Nucl. Instr. and Meth. A, 834, 46 
